\title{
Soil composition and plant genotype determine benzoxazinoid- mediated plant-soil feedbacks in cereals
}

\author{
Selma Cadot ${ }^{1,2,3} \odot$ | Valentin Gfeller ${ }^{2} \odot$ | Lingfei Hu${ }^{4} \odot$ | Nikhil Singh ${ }^{5}$ | \\ Andrea Sánchez-Vallet $^{6,7}$ ( ) | Gaétan Glauser ${ }^{8}$ ( ) | Daniel Croll ${ }^{5}$ ( | \\ Matthias Erb ${ }^{2}$ () | Marcel G. A. van der Heijden ${ }^{1}$ () | Klaus Schlaeppi ${ }^{1,2,3}$ ()
}

${ }^{1}$ Division of Agroecology and Environment, Agroscope, Zurich, Switzerland

${ }^{2}$ Institute of Plant Sciences, University of Bern, Bern, Switzerland

${ }^{3}$ Department of Environmental Sciences, University of Basel, Basel, Switzerland

${ }^{4}$ Zhejiang Provincial Key Laboratory of Agricultural Resources and Environment, Zhejiang University, Zhejiang, China

${ }^{5}$ Laboratory of Evolutionary Genetics, University of Neuchâtel, Neuchâtel, Switzerland

${ }^{6}$ Plant Pathology, Institute of Integrative Biology, ETH Zürich, Zürich, Switzerland

${ }^{7}$ Centro de Biotecnología y Genómica de Plantas (CBGP), Instituto Nacional de Investigación y Tecnología Agraria y Alimentaria (INIA), Universidad Politécnica de Madrid (UPM), Campus de Montegancedo UPM, Pozuelo de Alarcón (Madrid), Spain

${ }^{8}$ Neuchâtel Platform of Analytical Chemistry, University of Neuchâtel, Neuchâtel,

Switzerland

\section{Correspondence}

Klaus Schlaeppi, Department of Environmental Sciences, University of Basel, Bernoullistrasse 32, 4056 Basel, Switzerland.

Email: klaus.schlaeppi@unibas.ch

\section{Funding information}

This study was supported by the Swiss State Secretariat for Education, Research and Innovation (grant C15.0111 to ME, MvdH and $\mathrm{KS}$ ), and the University of Bern through the Interfaculty Research Cooperation 'One Health'. NK was supported by the Swiss National Science Foundation (grant 31003A_173265 to DC).

\begin{abstract}
Plant-soil feedbacks refer to effects on plants that are mediated by soil modifications caused by the previous plant generation. Maize conditions the surrounding soil by secretion of root exudates including benzoxazinoids (BXs), a class of bioactive secondary metabolites. Previous work found that a BX-conditioned soil microbiota enhances insect resistance while reducing biomass in the next generation of maize plants. Whether these BX-mediated and microbially driven feedbacks are conserved across different soils and response species is unknown. We found the BX-feedbacks on maize growth and insect resistance conserved between two arable soils, but absent in a more fertile grassland soil, suggesting a soil-type dependence of BX feedbacks. We demonstrated that wheat also responded to BX-feedbacks. While the negative growth response to BX-conditioning was conserved in both cereals, insect resistance showed opposite patterns, with an increase in maize and a decrease in wheat. Wheat pathogen resistance was not affected. Finally and consistent with maize, we found the BX-feedbacks to be cultivar-specific. Taken together, BXfeedbacks affected cereal growth and resistance in a soil and genotype-dependent manner. Cultivar-specificity of BX-feedbacks is a key finding, as it hides the potential to optimize crops that avoid negative plant-soil feedbacks in rotations.
\end{abstract}

\section{KEYWORDS}

maize, soil conditioning, wheat, root exudates, microbiome 


\section{1 | INTRODUCTION}

Plants modify their surrounding soil environment to optimize their performance and these soil modifications then affect the performance of other plants growing later in this soil. This phenomenon, where soil legacies from a previous plant generation modify the performance of the next plant generation, is well-known as plant-soil feedbacks (PSFs, van der Putten et al., 2013). The first plant generation may change physical, chemical and/or biological characteristics of the soil, a process often referred to as 'soil conditioning' (Bezemer et al., 2006). Such modifications of the soil condition then feedback on the next generation's growth, development and/or metabolism, and can influence defence and/or tolerance to biotic and abiotic stresses (Huberty, Choi, Heinen, \& Bezemer, 2020; Pineda, Kaplan, Hannula, Ghanem, \& Bezemer, 2020; Revillini, Gehring, \& Johnson, 2016; Zhao et al., 2021).

Negative PSFs are thought to occur because the previous plant generation enriched pathogens, released allelopathic compounds and/or reduced the availability of nutrients whereas promotion of beneficial symbionts and/or an enhancement of nutrient availability by the previous generation would result in positive PSFs (Bever, 2003; van der Putten et al., 2013). Therefore, PSFs represent important factors that determine the coexistence and diversity of plant communities in natural ecosystems (Bennett et al., 2017; Teste et al., 2017; van der Putten, 2017). For instance, PSFs regulate through mycorrhizal symbiont types the population structure of temperate forests (Bennett et al., 2017). Interestingly, not only the conditioning by the first plant generation, but also the interpretation of soil legacies' by the subsequent plant generation is key that PSFs promote plant diversity. Teste et al. (2017) revealed in Mediterranean shrublands that the nutrient-acquisition strategy of the response plants explained differential feedbacks to soil biota and thereby promoted local plant diversity. Besides in natural ecosystems, PSFs are very important in the agricultural context where the growth of the previous crop impacts the performance and yield of the following crop (Mariotte et al., 2018; Pizano, Kitajima, Graham, \& Mangan, 2019). Although, not specifically following the terminology of PSFs, farmers have recognized for a long time that certain sequences of crop plantings negatively affected their yields. Using dedicated crop rotations, farmers take advantage of positive while avoiding negative PSFs. For example, Brassica crops are specifically used in rotations, a cropping method referred to as biofumigation, because of their positive feedbacks on the following crops (Gimsing \& Kirkegaard, 2009). Brassicaceae plants produce secondary metabolites, that is, glucosinolates, that function as natural biocides to control fungal and oomycete pathogens and plant-parasitic nematodes (Brennan, GlazeCorcoran, Wick, \& Hashemi, 2020; Poveda, Eugui, \& Velasco, 2020). Coupling such practical knowledge with a fundamental ecological understanding is likely to offer further opportunities for harnessing PSFs in sustainable agriculture (Mariotte et al., 2018; Pineda et al., 2020). Possible applications of PSFs ranging from seed selection to agricultural biodiversity management, including fine-tuning the already widely used crop rotation systems.
PSFs often affect the next plant generation's defence against pests and pathogens. For example, soil conditioning by eight forb and grass species consistently suppressed aboveground infestations by Frankliniella occidentalis where these plants shaped species-specific soil microbiomes causing varying amplitudes of thrips resistance, secondary metabolite concentrations in leaves and plant growth (Pineda et al., 2020). These positive PSFs on resistance against thrips were not effective against the spider mites Tetranychus urticae, corroborating that differing plant traits shape specific soil legacies that then result in specific plant-insect feedbacks (Heinen, Biere, \& Bezemer, 2020). Similarly, PSFs improved plant protection against pathogens as for example in Arabidopsis thaliana against the aboveground pathogen Pseudomonas syringae (Yuan et al., 2018) or the root pathogen Pythium ultimum shown with Chrysanthemum by Hannula, Ma, Pérez-Jaramillo, Pineda and Bezemer (2020). Pest and pathogen control in PSFs may function via attraction or enrichment of beneficial soil microbes or their shifts in the whole microbial community and they function in positive feedbacks (Bakker, Pieterse, de Jonge, \& Berendsen, 2018; Berendsen et al., 2018).

Plants secrete considerable amounts of bioactive molecules from their roots into the rhizosphere and this root exudation is responsible for a large part of belowground soil conditioning (Bever, Platt, \& Morton, 2012). Exudation directly benefits a plant by enhancing nutrient availability (Jones \& Darrah, 1995), suppressing competitors by allelopathy (Inderjit \& Duke, 2003) and importantly, it is largely responsible for the plant's influence on the rhizosphere microbiota, which then indirectly benefits the plant (Sasse, Martinoia, \& Northen, 2018). The rhizosphere microbiome confers health benefits to the plant (Berendsen, Pieterse, \& Bakker, 2012), by mechanisms such as direct protection (antibiosis), niche competition with pathogens for resources or by enhancing the immune response from the plant via induced systemic resistance (ISR, Pieterse et al., 2014). Root exudate metabolites such as coumarins or benzoxazinoids are known to selectively structure the rhizosphere microbiome, and these changes have been linked to improved plant health (Hu et al., 2018; Stringlis et al., 2018).

Plants from the Poaceae family, including important cereals such as wheat, maize and rye, produce and exude benzoxazinoids (BXs) from their roots to the surrounding soil. BXs are a family of bioactive secondary metabolites with allelopathic properties (Macías, Marín, Oliveros-Bastidas, \& Molinillo, 2009; Schandry \& Becker, 2020), direct protective functions against herbivore insects (Niemeyer, 2009; Wouters, Blanchette, Gershenzon, \& Vassão, 2016; Zhou, Richter, \& Jander, 2018) and fungal or bacterial pathogens by toxicity (Martyniuk, Stochmal, Macías, Marín, \& Oleszek, 2006; Schalchli et al., 2012), or by suppressing the virulence of pathogens (Sicker, Frey, Schulz, \& Gierl, 2000). BXs function in selectively structuring the root and rhizosphere microbiomes (Cadot et al., 2021; Cotton et al., 2019; Hu et al., 2018; Kudjordjie, Sapkota, Steffensen, Fomsgaard, \& Nicolaisen, 2019), not only via antagonistic functions of BXs described above, but also through attraction, as evidenced by the positive chemotactic response of Pseudomonas putida to locate maize roots (Neal, Ahmad, Gordon-Weeks, \& Ton, 2012). Of note, the 
selective recruitment of $P$. putida by BXs may promote plant health, as this strain is capable of triggering ISR in maize (Neal \& Ton, 2013).

In a previous study, we demonstrated that the secretion of BXs conditions the surrounding rhizosphere microbiota that then drives PSFs on the next plant generation (Hu et al., 2018). These feedbacks become visible when comparing the soil variant that was conditioned by $\mathrm{BXs}(B X+$ soil) with the control soil variant that was not conditioned by $B X s$ ( $B X$ - soil). Feedbacks on a new generation of maize plants included enhanced insect resistance and reduced plant growth for plants grown on $\mathrm{BX}+$ soil. The maize plants responded physiologically with an increase in defence hormone levels and an upregulation of defence marker genes. Sterilization and complementation experiments demonstrated that these feedbacks were driven by the soil microbiota. We refer to these BX-mediated and microbially driven PSFs hereafter as 'BX-feedbacks' for simplification.

A number of follow-up research questions related to agricultural implications emerged from our initial mechanistic study on BXfeedbacks (Hu et al., 2018), which was limited to maize and examined only a single soil. First, if BX-feedbacks would be relevant in agriculture, they should also occur in other soils. This seemed possible as it is well-known that PSF happen in different soil types and with different microbiota pools (Bergmann et al., 2016; Smith-Ramesh \& Reynolds, 2017), and we also knew that different soils have BXsensitive microbes (Cadot et al., 2021). Therefore, we asked (a) whether BX-feedbacks would also occur in other soils. Second, to be relevant in agriculture, BX-feedbacks should also be manifested in crop rotations, a common cropping practice. It seemed plausible, as PSF responses varied between plant species and cultivars (Heinen et al., 2020; Kutáková, Herben, \& Münzbergová, 2018; Wagg, Boller, Schneider, Widmer, \& van der Heijden, 2015). While we knew that BX-feedbacks occur on maize after maize (Hu et al., 2018), it was unknown if another crop would respond to a BX-conditioned soil. As wheat often follows maize in crop rotations in Europe we asked (b) whether wheat would also respond to BX-feedbacks. Thirdly, so far we have tested BX-feedbacks only on insect resistance $\mathrm{Hu}$ et al., 2018) but crops in the field are exposed to a variety of other biotic stresses. Therefore, because of reported trade-offs between pathogen and insect defence strategies (Erb et al., 2011; Zhu et al., 2018), we asked (c) whether BX-feedbacks would also affect pathogen defence. In this study, we addressed these three open research questions and we showed that BX-feedbacks are also effective on wheat and that they function in soil and cultivar-dependent manner.

\section{2 | METHODS}

\subsection{Overview of BX-feedback experiments}

This study comprised six complementary experiments, all investigating BX-feedbacks comparing BX-conditioned (BX+ soil) with nonconditioned soil variants ( $B X-$ soils; see below). To answer the first research question, whether $B X-$ feedbacks depended on soil, we setup the 'Experiments 1 and 2'. Each experiment was designed to study BX-feedbacks in one other soil and we measured BX-feedbacks on growth and insect defence of wild-type maize. In 'Experiment 1' we tested a loam soil from a field trial in 'Reckenholz' near Zurich and in 'Experiment 2' we tested a silt loam soil sampled from the grassland site 'Q-matte' nearby Bern (both Switzerland). In both experiments, we included soil from 'Changins' as a positive control because BXfeedbacks were originally discovered in this clay loam soil $\mathrm{Hu}$ et al., 2018). 'Reckenholz' and 'Q-matte' soils were used since they originate from other geographical areas and because of their different biogeochemical characteristics compared to 'Changins' soil (Table S2). With the 'Reckenholz' soil, we further compared freshly conditioned soil with soil that was conditioned a year before. The goal was to learn, whether BX-feedbacks remain preserved upon storage of the conditioned soils for up to 1 year at $4^{\circ} \mathrm{C}$ in the cold room.

With the 'Experiments 3 to 5' we investigated the second and third research questions, whether BX-feedbacks also occur on wheat growth and defence against insect and pathogen. We performed these experiments mainly with 'Changins' soil because the feedbacks on maize were originally discovered with this soil (Hu et al., 2018). In 'Experiment 3' we examined BX-feedbacks on growth (biomass) and physiology (chlorophyll content, hormones) testing two wheat cultivars as response plants. For comparison, we included Reckenholz soils batches as used in the first experiment. 'Experiment 4' and 'Experiment 5 ' were designed to specifically test BX-feedbacks on insect and pathogen resistance, respectively. We conducted performance assays with the herbivore Spodoptera frugiperda with plants of two wheat cultivars in 'Experiment 3'. With 'Experiment 5' we studied resistance against the wheat pathogen Zymoseptoria tritici using one of the two cultivars.

Finally, 'Experiment 6' was conducted with potting soil to uncover if the genotype-dependent BX-feedbacks on maize shoot biomass and insect resistance occur during the conditioning or the feedback phase. See Appendix S1 for the detailed experimental setups of each of these six experiments.

\subsection{Feedback experiments with conditioned soils}

Conditioning functioned by growing the wild-type (WT) line B73, which secreted BXs to the surrounding soil (Hu et al., 2018; Maag et al., 2014). The control soil variant was prepared by growing the near-isogenic mutant line $b \times 1$ (B73), which is compromised in the biosynthesis and secretion of BXs (Maag et al., 2014). Soil variants conditioned by the growth of WT maize are referred to as $B X+$ soil, and soil variants, where the mutant $b \times 1$ was grown, are referred to as $B X-$ soil. Soil conditioning was standardized, by growing the B73 and bx1 lines for 3 months in a complete randomized block design.

For this study, we used several batches of conditioned soils and they were either prepared in the field or in greenhouse experiments (Table S1, detailed below). We have previously described the details of the field experiments, of which we collected the conditioned soil batches 'Changins 2016' (Hu et al., 2018) and 'Reckenholz 2016' 
(Cadot et al., 2021). The conditioned soil batch 'Reckenholz 2015' (Table S1) was harvested from a field experiment, which we performed in 2015 with the same setup as the field experiment in 2016. See Appendix S1 for the details of the field experiment conducted at Agroscope Reckenholz in 2015. We needed to condition additional soils for further experiments (Table S1) and this was done in the greenhouse, knowing that this type of conditioning works, too ( $\mathrm{Hu}$ et al., 2018). The details of the greenhouse experiments to condition the soil are documented in Appendix S1.

Prior to their use for feedback experiments, all batches of conditioned soils were sieved to $1 \mathrm{~cm}$ and at the same time mixed with autoclaved quartz sand in a $1: 4$ sand to soil proportion ( $20 \%$ by volume). Importantly, the metal sieve was always sterilized with $70 \%$ $\mathrm{ETOH}$ between $\mathrm{BX}+$ and BX- soil variants to avoid the transfer of soil and microbes.

We have previously reported the chemical and physical characteristics from the clay loam soil in Changins and loam soil from Reckenholz (Cadot et al., 2021). We also determined the soil characteristics of the silt loam soil from Q-Matte in the same laboratory using the same certified methods (Labor für Boden- und Umweltanalytik, Eric Schweizer AG, Thun, Switzerland). Soil texture classes were determined using the online tool of the Natural Resources Conservation Service for Soils at the Unites States of Department of Agriculture. See Table S2.

\section{3 | Chlorophyll measurements}

Wheat chlorophyll content was measured in Experiments 3 and 4 on the second fully opened leaf using a Soil Plant Analysis Development SPAD-502 m (Minolta Camera Co., Japan), at around $1 \mathrm{~cm}$ below the tip on two plants per pot, and the values were averaged for each pot for analysis.

\section{4 | Phytohormone analysis}

To obtain insights into the plant defence status, we measured the following plant hormones (or precursors) in wheat samples of Experiment 3 salicylic acid (SA), oxophytodienoic acid (OPDA), jasmonic acid (JA), jasmonic acid-isoleucine (JA-lle) and abscisic acid (ABA). The frozen leaf material was ground using mortar, pestle and liquid nitrogen, transferred to $1.5 \mathrm{ml}$ microcentrifuge tubes and precisely weighed. Hormone analysis was performed according to (Glauser, Vallat, \& Balmer, 2014). The resulting hormone concentrations were then standardized according to initial leaf weight.

\subsection{Insect assays}

Second (Experiment 6) or third (Experiment 1) instar S. frugiperda caterpillars were used to assess maize insect resistance as described in $\mathrm{Hu}$ et al. (2018). Briefly, they were weighed and selected for similar weights before starting the feeding assay. They were placed into transparent plastic cups ( $4 \mathrm{~cm}$ height and $3.5 \mathrm{~cm}$ diameter) that have perforated plastic lids. The youngest fully developed leaves of individual maize plants were used for feeding. Leaves were placed as ca. $3 \mathrm{~cm}$ portions (excluding the leaf tip) into the cups with some moisture. These leave segments were daily replaced by new portions of the same leave throughout the assay. Caterpillar mass was determined 3 days after the start of the assay and the growth rate was calculated (Growth rate $=$ [Weight at day $X$ - initial weight] / initial weight).

In Experiment 3 we evaluated wheat insect resistance using second instar caterpillars of Spodoptera littoralis. They were weighed before starting the feeding assay and two to three caterpillars were placed in small perforated plastic boxes. Two leaves per wheat pot were fed portioned by pieces for 1 week. Leaf pieces were replaced in the box and moisturized after 3 days and then every 2 days as the caterpillars grew bigger. Caterpillars were weighed at 4, 5 and 7 days (D) of feeding and the growth rate was calculated (formula as above).

\section{6 | Pathogen assay}

In experiment 4, we tested for eventual differences in pathogen resistance of the wheat cultivar Drifter when growing on ' $\mathrm{BX}+$ ' or ' $\mathrm{BX}-$ soils' using the fungus $Z$. tritici (strain 3D7). The second fully unrolled leaf of 3-week-old plants was spray-infected with $10 \mathrm{ml}$ of blastospores following a standard infection protocol (Singh, Badet, Abraham, \& Croll, 2021). The inoculated plants were kept at $100 \%$ relative humidity and $21^{\circ} \mathrm{C}$ for 2 days, before going back to initial growth conditions (see above). Five additional plants were left uninoculated as controls. The inoculated leaves were harvested 21 days postinfection for counting pycnidia (reproductive structures of the fungus attesting successful fungal multiplication in the host tissue) using the software ImageJ.

Fungal biomass in the inoculated leaves was quantified on the same plant material by quantitative PCR (qPCR), using the fungal strain-specific forward primer sequence $5^{\prime}$ cgacatcggttcagagatggaa' 3 and reverse primer sequence $5^{\prime}$ 'gtaccttcgattcgtgcggt 3 , and the plant $18 \mathrm{~S}$ forward primer sequence $5^{\prime}$ cgcagcaaatcccacgg 3 and reverse primer sequence $5^{\prime}$ gcgcagcttcttccactttgac'3. Genomic DNA was extracted with a DNA easy plant kit (Qiagen, Germany) with minor modifications (samples were ground on the grinding machine Qiagen Tissue Lyser II, $2 \times 30 \mathrm{~s}$ at setting 30 , incubated in the final step for $15 \mathrm{~min}$ at $65^{\circ} \mathrm{C}$ and the DNA was eluted in a volume of $150 \mathrm{ul}$ ). Realtime $\mathrm{qPCR}$ reactions were then performed according to Meile et al. (2018) and Barrett et al. (2021). We used the $\Delta \Delta \mathrm{C}_{\mathrm{T}}$ method to express the fungal biomass relative to the plant leaf signal $\left(E^{\wedge} \Delta C_{T}\right.$ (Fungus, control - infected)/ $\mathrm{E}^{\wedge} \Delta \mathrm{C}_{\mathrm{T}}$ (Plant, control - infected).

\subsection{Statistical analyses}

Data analysis was performed in $\mathrm{R}$ (version 3.5.1, R Development Core Team, 2017) using R Markdown and the package 'ggplot2' for plotting 
(Wickham, 2016). For each experiment, we provide the R markdown code, the raw data and functions required for replication of the analysis as .zip archives at https://github.com/PMI-Basel/Cadot_et_al_

(a)

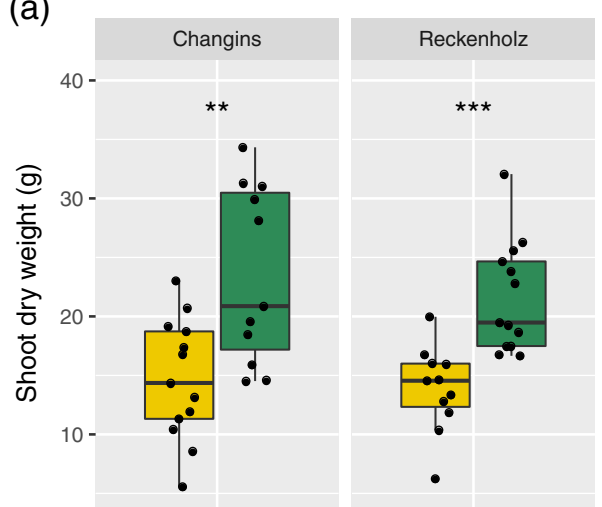

(b)

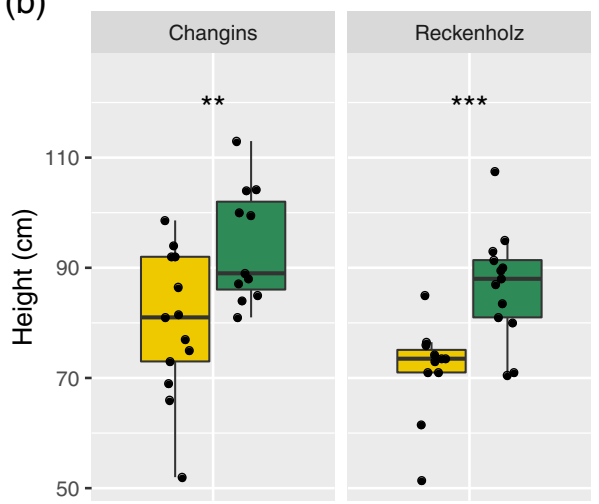

(c)

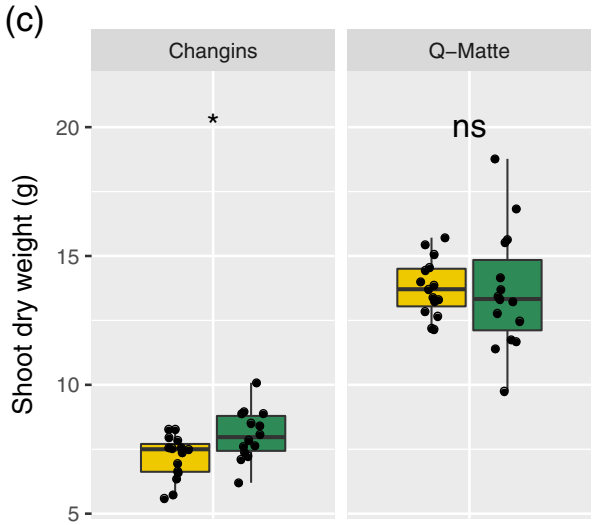

(d)

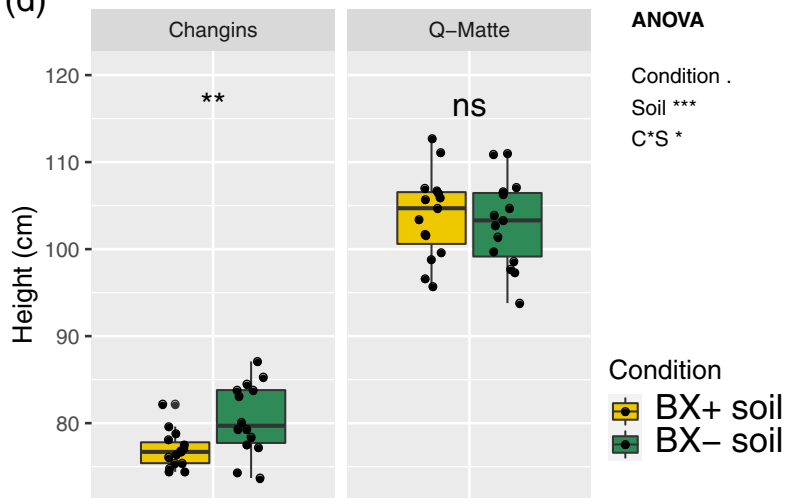

ANOVA

Condition *** Soil * C*S n.s.

ANOVA Condition . Soil ${ }^{* \star *}$ $\mathrm{C}^{*} \mathrm{~S}^{*}$ wheat_feedbacks. The statistical results (incl. the models used) and the design of Experiments 1 to 6 are documented in the supplementary Data S1-S6 (R Markdown reports).

As a general approach, we inspected the data whether they satisfied normality assumptions using residual plots following (Fahrmeir, Kneib, Lang, \& Marx, 2013). Most one-time point data (biomass, height, chlorophyll, hormones) was examined with ANalyses Of VAriance assessing the effect of conditioning (BX+vs. BX-) and combinations with effects of soil (Experiment $1 \& 2$ ), wheat line (Experiment $3 \& 4$ ), or genetic background (Experiment 6). Pair-wise T-tests were performed in a post-hoc manner (Experiment 1, 2, 3 and 4) or using Tukey HSD tests (Experiment 6). Data with multiple time points (4x SPAD in Experiment 4; 3x caterpillar in Experiment 5) were analysed with linear mixed-effect models (LMM) using the package 'Ime4' (Bates, Mächler, Bolker, \& Walker, 2015). Pycnidia counts from Experiment 5 were analysed with a generalized linear model (glm) using a quasipoisson distribution model.

\section{$3 \mid$ RESULTS}

\section{1 | BX-feedbacks on maize are soil-dependent}

To answer the first question of this study - Do BX-feedbacks on maize also exist in other soils? - we tested with two experiments whether the BX-feedbacks, as observed in formerly used Changins soil $\mathrm{Hu}$ et al., 2018), also occur in two other soils. Reckenholz and Q-Matte are loam and silt loam soils, respectively and have markedly different physicochemical characteristics compared to clay loam soil in Changins (Table S2). We measured shoot height, shoot biomass and insect of maize plants growing on 'BX+' and 'BX-' soils. In the first experiment, we compared Reckenholz relative to Changins soil. Consistent with the Changins soil, we also found for the Reckenholz soil a significantly lower shoot biomass (Figure 1a) and shoot height (Figure 1b) when plants were grown on 'BX+' compared to 'BX-' soil variants (Data S1, documents the statistical analyses). Biomass reduction on ' $\mathrm{BX}+$ soil' accounted for $-43.7 \%$ in the Reckenholz soil, which is similar as for the Changins soil (-46.1\%). Testing insect resistance, we found that $S$. frugiperda caterpillars grew at a reduced rate in Reckenholz (-32.2\%) and Changins $(-29.6 \%)$ soils when feeding on leaves of maize plants that were grown on ' $\mathrm{BX}+$ ' compared to ' $\mathrm{BX}-$-'

FIGURE 1 BX-feedback on maize growth. Maize plants were grown on 'BX+' and 'BX-' variants of Changins soil as a control and compared to Reckenholz $(a, b)$ and in a separate experiment to QMatte soil (c,d). Shoot biomass (a, dry weight) and shoot height (b) was recorded after 10 weeks. Data S1 documents the statistical analyses in detail. The Q-matte experiment was harvested after 9 weeks and 3 days measuring shoot biomass (c, dry weight) and shoot height (d); Data S2 for statistic details. The ANOVA results (model: $\sim$ condition [C] ${ }^{*}$ soil [S]) are reported next to the figure and the pair-wise T-test results inside the panels (Significance code: $p<.001^{* * *} ; p<.01^{* *}, p<.05^{*}$; not significant = 'n.s.') [Colour figure can be viewed at wileyonlinelibrary.com] 
soil variants (Figure 2, Data S1). In the second experiment, comparing Q-Matte with Changins soil, we confirmed the feedbacks in Changins soils but we did not find feedbacks on shoot biomass (Figure 1c) or height (Figure 1d) for plants grown on 'BX+' compared to 'BX-' variants of the Q-Matte soil (Data S2). Insect resistance could not be tested in the second experiment.

We found earlier that BX-feedback soils remained functional after a winter in the field (Hu et al., 2018) and here we tested the technical question, whether BX-feedbacks could also be preserved by storing the conditioned soils in the cold room. The soil batch 'Reckenholz $\left(4^{\circ}\right)$ ' was stored for 1 year in the cold room and then assessed in parallel with fresh Reckenholz soil for BX-feedbacks. Similar to the fresh soil batch, we found significantly lower shoot biomass (Figure S1a), shoot height (Figure S1b) and caterpillar growth rates (Figure S1c) in the 'technically overwintered' batch (Reckenholz $4^{\circ}$; Data S1).

Taken together, BX-feedbacks on maize were in agreement with earlier findings (Hu et al., 2018), occurred also in Reckenholz soil and in soil that was stored for 1 year in the cold room. However, no feedbacks were detected on the more fertile Q-Matte soil, revealing that BX-feedbacks are soil-dependent.

\subsection{Wheat biomass is reduced by BX-feedbacks}

To answer the second question of this study - Do BX-feedbacks also occur in wheat? - we performed different experiments to examine feedback responses (a) on growth and physiology, (b) insect resistance and (c) pathogen resistance. We included two wheat cultivars in most of these experiments to assess whether responses are cultivardependent.

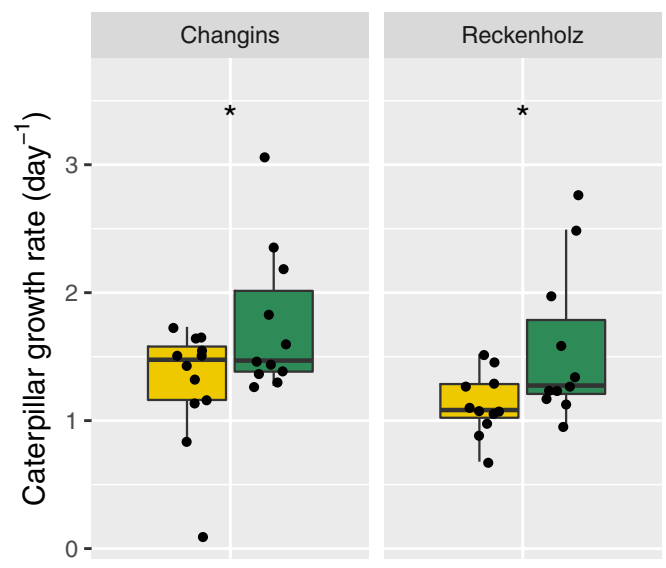

ANOVA

Condition **

Soil n.s.

C*S n.s.

Condition

아 BX+soil

BX-soi

FIG URE 2 BX-feedback on maize insect resistance. Maize plants were grown on ' $\mathrm{BX}+$ ' ' and ' $\mathrm{BX}-$ ' variants of Changins soil as a control and in two batches of Reckenholz soil. Caterpillar performance of Spodoptera frugiperda was measured with leaves of 9-week-old plants. Data S1 documents the statistical analyses in detail. The ANOVA results (model: $\sim$ condition $[\mathrm{C}]^{*}$ soil $[\mathrm{S}]$ ) are reported next to the Figure and the pair-wise T-test results inside the panels (Significance code: $p<.001^{* * *} ; p<.01^{* *}, p<.05^{*}$; not significant $=$ 'n.s.') [Colour figure can be viewed at wileyonlinelibrary.com]
As indications for plant growth, we measured fresh and dry biomass weight of the two cultivars Drifter and Fiorina when grown on 'BX+' and 'BX-' soil variants. We found a significantly lower fresh (Figure $3 a$ ) and dry (Figure $3 b$ ) biomass when wheat was grown on 'BX+' compared to 'BX-' soil variants. Shoot fresh weight was reduced in both wheat cultivars and both soils (Drifter and Fiorina, 10.1 and $-12.2 \%$ in Changins and -9.0 and $-11.9 \%$ in Reckenholz soil, respectively). Similarly shoot dry weight was mostly reduced on 'BX+' soil variant (Drifter and Fiorina, -1.3 and $0 \%$ in Changins soil, -4.0 and $-1.9 \%$ in Reckenholz soil). These results were supported by factorial ANOVA but not pairwise tests except for dry biomass of Drifter on Reckenholz soil (Figure 3b, Data S3) We concluded that BX-feedbacks negatively impacted the growth of the two wheat cultivars in both soils.

We also tested if BX-feedbacks affected wheat physiology and therefore, we approximated leaf chlorophyll content with SPAD measures between the third and the sixth week of wheat growth (Figure S2). Although plant chlorophyll content varied

(a)
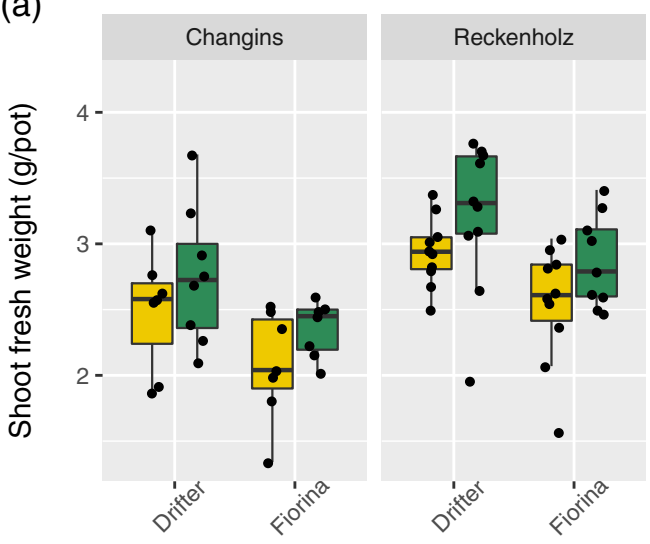

ANOVA

Condition ** Wheat line ${ }^{* * *}$ C*WL n.s.

(b)

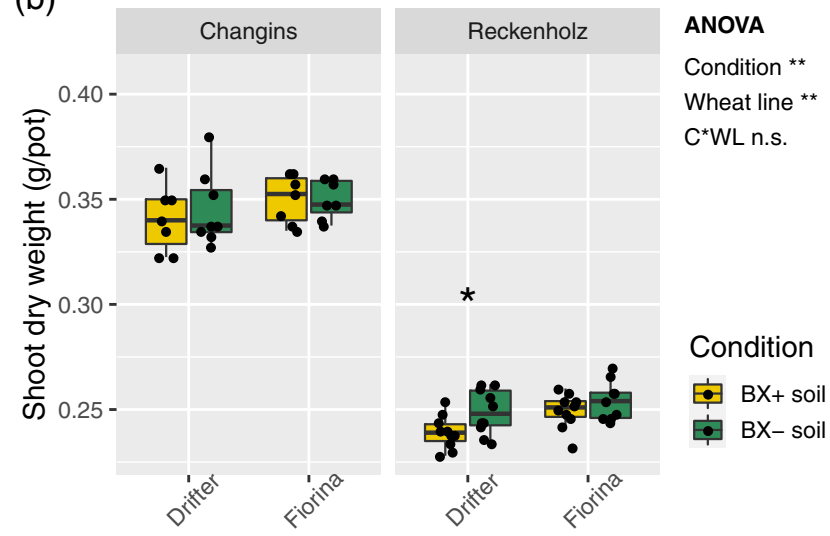

FIGURE 3 BX-feedback on wheat growth. Wheat plants were grown on 'BX+' and 'BX-' variants of Changins and Reckenholz soils. Shoot biomass was measured as (a) fresh and (b) dry weight for the two wheat lines Drifter and Fiorina after 6 weeks of growth. Data S3 documents the statistical analyses in detail. The ANOVA results (model: $\sim$ condition $[\mathrm{C}]{ }^{*}$ wheat line $[\mathrm{WL}]$ ) are reported next to the Figure and the pair-wise T-test results inside the panels (Significance code: $p<.001^{* * *} ; p<.01^{* *}, p<.05^{*}$; not significant = 'n.s.') [Colour figure can be viewed at wileyonlinelibrary.com] 
more strongly with time, between the soils and between wheat varieties, we noticed a significant effect of BX-conditioning with generally higher chlorophyll content in wheat plants grown on 'BX+' compared to 'BX-' soils (Data S3). This effect was most apparent in week 4 with particularly reduced chlorophyll contents in Fiorina plants grown on 'BX-' soils $(-20.4 \%$ in Changins and $-6.2 \%$ in Reckenholz soil). Hence, BX-feedbacks positively affected wheat physiology by transiently enhancing leaf chlorophyll in one cultivar.

For further physiological insights, we measured the classical plant defence hormones SA, OPDA, JA, JA-lle and ABA in plants grown on ' $\mathrm{BX}+$ ' vs. ' $\mathrm{BX}-$ ' soil variants. With the exception of OPDA, hormone levels differed significantly between the cultivars with Drifter generally having higher levels of SA, JA and JA-Ile than Fiorina (Figure S3, Data S3). For ABA, however, concentrations were higher in Fiorina compared to Drifter. Soil pre-conditioning by BXs did not affect any of the plant hormone concentrations. Thus, BX-feedbacks do not directly alter constitutive leaf hormone levels in wheat.

In summary, BX-feedbacks also occurred on wheat growth with lower biomass and effects on physiology with a generally higher shoot chlorophyll content but no effects on plant defence hormone levels.

\subsection{Genotype of the response plant explains variations in BX-feedbacks}

In our earlier work, we already found that BX-feedbacks on maize growth were genotype-specific (Hu et al., 2018). Because we had studied the feedbacks of B73 plants on their B73 conditioned soils and those of W22 plants on their conditioned soils, it remained unclear whether the cause for the effects on growth occurred during the conditioning or during the feedback phase. To close this gap, we performed here an experiment where we grew B73 plants but on W22 conditioned soils. Consistent with the earlier findings in Changins soil, we measured also in potting soil a significantly lower shoot biomass (Figure 4a) and lower insect performance (Figure 4b) for the $B 73$ response plants on their own conditioned ' $\mathrm{BX}+$ ' and 'BX-' soil variants (Data S4). When growing B73 as response plants on the soil variants conditioned by W22 lines, we found the same negative growth and insect phenotypes as on B73 conditioned soils (Figure 4) while this was not the case for W22 as response genotype (Hu et al., 2018). Hence, we identified the feedback and not the conditioning phase as causal and we concluded that the different genotypes of the response plant explain the different findings.

\section{4 | BX-feedbacks on wheat insect resistance are cultivar-specific}

As the genotype-dependent BX-feedbacks on maize specifically enhanced insect resistance, we also examined the two wheat cultivars Drifter and Fiorina for eventual BX-feedbacks when grown on 'BX+' (a)

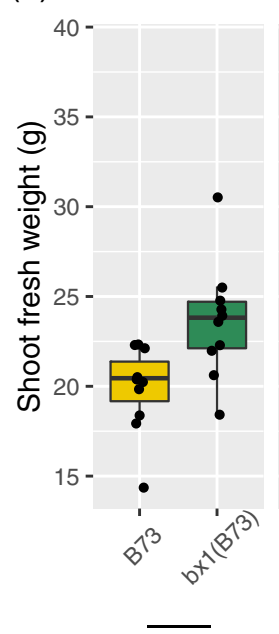

B73

(b)

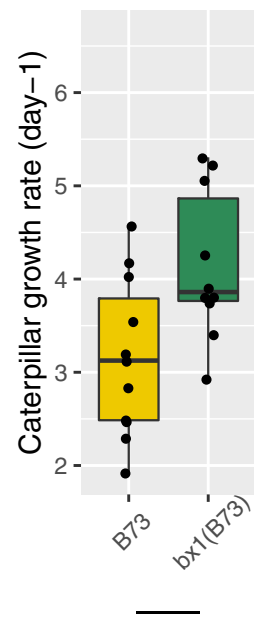

B73

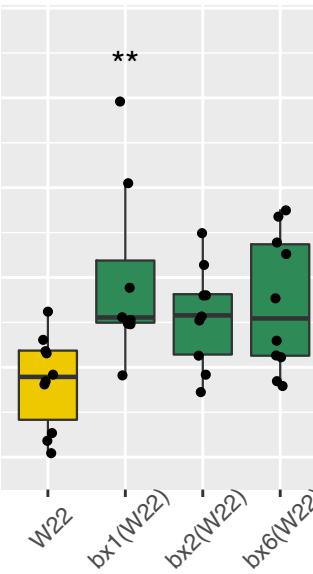

B73

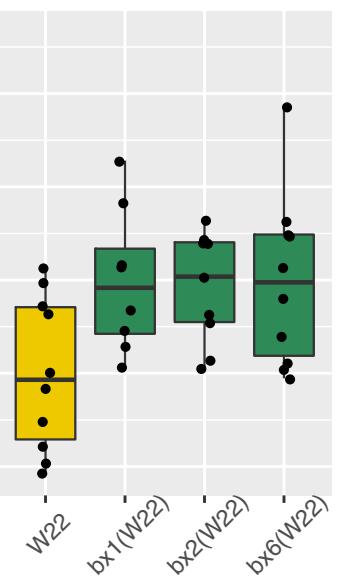

B73
ANOVA

conditioning variables $\mathrm{BX}$ ***

gen. background n.s. $B X^{\star} \mathrm{GB}$ n.s.

- Conditioning

- Response conditioning variables $B X$ **

gen. background n.s. $B X^{\star} \mathrm{GB}$ n.s.

\section{Condition}

E⿵冂⿱一口犬 $B X+$ soil

E바 BX-soil

- Conditioning

FIGURE 4 Genetics of BX-feedback on maize. Potting soil was conditioned with $\mathrm{B} 73$ and $b \times 1$ (B73) as well as with W22, bx1(W22), $b \times 2(\mathrm{~W} 22)$ and $b \times 6(\mathrm{~W} 22)$ followed by a feedback phase with only B73 plants $(n=8-11)$. Ten-week-old plants were utilized for measuring (a) shoot biomass (fresh weight) and (b) Spodoptera frugiperda performance. Data S4 documents the statistical analyses in detail. The ANOVA results (model: $\sim \mathrm{BX}$ condition $[\mathrm{BX}]^{*}$ genetic background condition [GB]) are reported next to the Figure and the pair-wise Tukey-test results inside the panels (pair-wise comparison with wildtype, significance code: $p<.01^{* *}, p<.05^{*}$; not significant $=$ 'n.s.') [Colour figure can be viewed at wileyonlinelibrary.com]

and 'BX-' soil variants. ANOVA analysis indicated that the two cultivars behaved differently in the two soil variants (Data S5). While insect growth rates were unaffected by BX-conditioning in Drifter, they were significantly higher on Fiorina when growing on ' $\mathrm{BX}+$ soil' (Figure 5a). This suggested that BX-feedbacks reduced the insect resistance of the wheat cultivar Fiorina while Drifter, the cultivar with generally higher defence hormone levels, was unaffected in its insect resistance. 
(a)

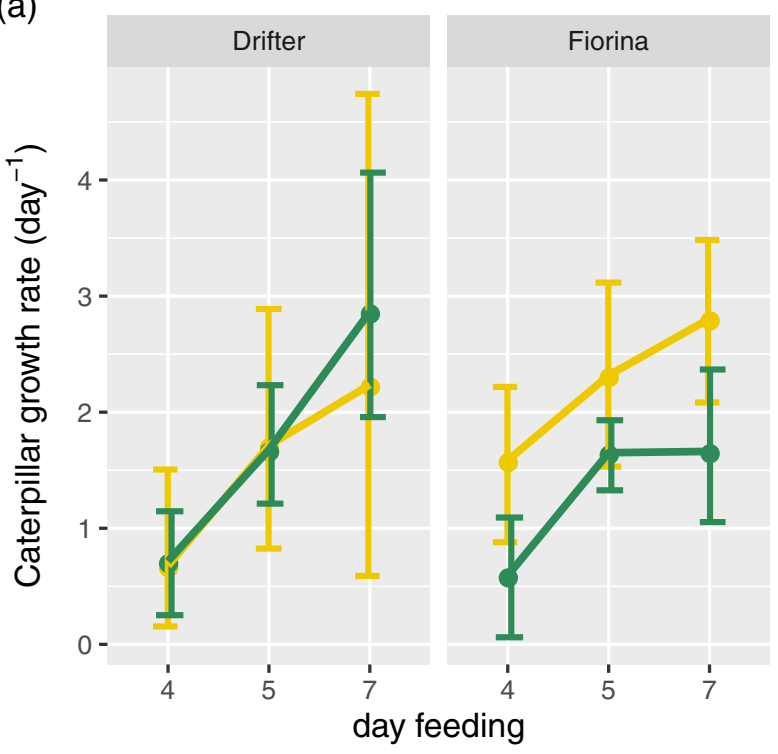

LMER

Condition n.s.

(Condition for subset Fiorina: *)

Wheat line n.s.

$\mathrm{C}^{*} \mathrm{WL}$. (b)

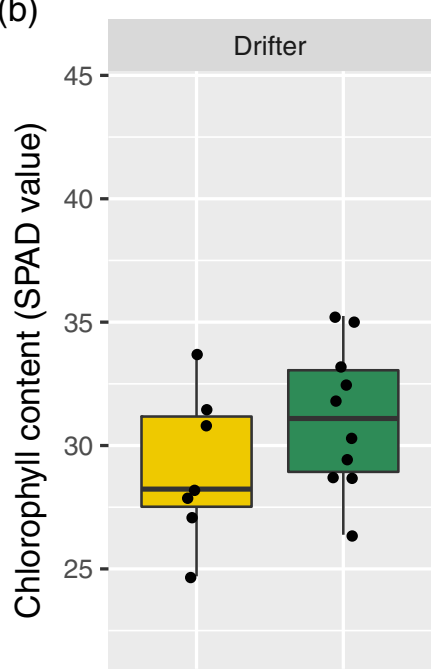

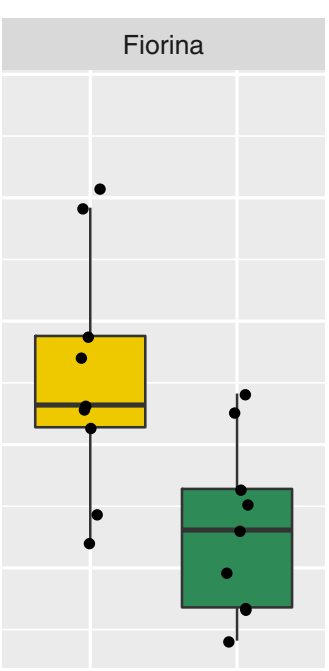

ANOVA

Condition .

(Condition for subset Fiorina: **) Wheat line n.s.

$\mathrm{C}^{*} \mathrm{WL}$ **
FIGURE 5 BX-feedback on wheat insect resistance and chlorophyll content. Wheat plants from cultivar Drifter and Fiorina were grown on 'BX+' and 'BX-' variants of Changins soil. (a) Caterpillar performance of Spodoptera littoralis fed with leaves of 5 -weeks-old plants was measured after 4, 5 and 7 days of feeding; and (b) leaf chlorophyll content (SPAD values) was measured on 6-weeks-old plants. Data S5 documents the statistical analyses in detail. The LME (model: day_feeding * condition [C] * wheat_line [WL], random factor $=$ individual) for (a) and ANOVA (model: condition [C] * wheat_line [WL]) for (b) results are reported next to the figure (significance code: $p<.001^{* * *} ; p<.01^{* *}, p<.05^{*}$; $p<.1$ '.; 'n.s.' = not significant) [Colour figure can be viewed at wileyonlinelibrary.com]
To confirm the BX-feedbacks on leaf chlorophyll content in the previous experiment, we repeated the SPAD measurements. Consistently with the previous results (Figure S2) we found again significantly reduced chlorophyll contents $(-20.8 \%)$ in Fiorina plants when grown on 'BX-' soils (Figure 5b, Data S5). And, as indicated by the significant interaction term of the ANOVA, there was no effect on Drifter plants. It appears that enhanced chlorophyll content, as a positive BX-feedback, presents a cultivar-specific response.

Overall, we conclude that BX-feedbacks on wheat are cultivarspecific as evidenced by the insect resistance and shoot chlorophyll assays.

\section{5 | No detectable impact of BX-feedbacks wheat pathogen resistance}

To evaluate whether BX-conditioning would also affect wheat pathogen resistance, we tested feedbacks to inoculations with $Z$. tritici.
There was no significant difference in pycnidia counts of plants growing on 'BX+' or 'BX-' soils (Figure S4a, Data S6). Plants growing on ' $B X+$ soil' tended to have higher numbers of pycnidia compared to plants growing on 'BX-' or the control soil). We also quantified $Z$. tritici in the infected leaf using qPCR, but pathogen abundance did not differ neither between the two BX conditions nor to the control soil (Figure S4b, Data S6). Taken together, we found no evidence that the BX-feedbacks impact the resistance of the wheat cultivar Drifter to the pathogen Z. tritici.

\section{4 | DISCUSSION}

BX-mediated microbial feedbacks influenced maize growth and insect resistance in one soil (Hu et al., 2018). Here, we explored these BXfeedbacks more broadly, asking first, whether they also occur in different soils and secondly, whether they also influence wheat as a typical crop following maize in European rotation schemes. We 
performed the latter experiments with two wheat cultivars, because our earlier maize work had revealed genetic differences in responsiveness to BX-feedbacks.

\section{1 | BX-feedbacks are soil dependent}

Consistent with our earlier work in soil from Changins (Hu et al., 2018), we also find BX-feedbacks on maize growth and insect resistance in the tested Reckenholz soil (Figures 1 and 2, Data S1). The feedbacks were of similar strength and in the same direction with reduced shoot biomass, lower shoot height and enhanced insect resistance for maize plants grown on 'BX+' compared to 'BX-' soil variants. Using sterilization we had demonstrated earlier that the differential feedbacks of maize growing either on 'BX+' and ' $\mathrm{BX}-$ ' variants of the Changins soil were driven by the microbiota ( $\mathrm{Hu}$ et al., 2018). We had also shown that the root and rhizosphere bacterial and fungal communities of the maize plants, which conditioned these soil variants (WT -> 'BX+', bx1 -> 'BX-'), differed in their composition. Therefore, it is tempting to assume that $B X$ exudation also changes the microbiota in the Reckenholz soil and that different microbiotas in 'BX+' and 'BX-' soil variants explain the differential feedbacks. While we have no experimental proof for the second part of the assumption, we found differences in community composition analysing the root and rhizosphere microbiotas of the maize lines, which conditioned the 'BX+' and 'BX-' variants of the Reckenholz soil, too (Cadot et al., 2021). Of note, these microbiota analyses were performed on the exact same plants, which conditioned the 'BX+' and ' $\mathrm{BX}-$ ' variants of the Reckenholz soil tested in this study. In Cadot et al. (2021) we investigated the general structuring of BX exudation on the root and rhizosphere microbiota and we found little overlap among BX-sensitive microbes in different field soils. This implies that different sets of BX-sensitive microbiotas can trigger similar feedback effects and that there is a functional overlap among them in different soils.

With this study, we now know that BX-feedbacks on maize function in the clay loam soil Changins and the loam soil Reckenholz, but not the silt loam soil Q-Matte. As the maize plants grew best in this soil (Figure 2), we consider Q-Matte as the most fertile among the tested soils. Given this first evidence that BX-feedbacks lose their effectivity at higher soil fertility, further work specifically investigating the impact of soil nutrients on BX-feedbacks is required. To what extent the feedbacks are affected by different soil histories (arable vs. grassland) also remains to be determined. Finally, further work is necessary to clarify the range of effective BX-feedbacks across various soil types and soil texture classes. A systematic examination with conditioning ' $\mathrm{BX}+$ ' and ' $\mathrm{BX}-$-' variants in fields of different soil types will present a tremendous effort, diverse soil types could be collected and then conditioned in the greenhouse to work out the impact of specific biogeochemical properties on BX-feedbacks. Ultimately, understanding the context dependency of such BX-feedbacks appears important, hypothesizing that these feedbacks do not only occur under controlled greenhouse but also under realistic agricultural conditions.

\section{2 | BX-feedbacks are preserved at $4^{\circ} \mathrm{C}$ for at least 1 year}

We knew that BX-feedbacks are preserved over a winter period in the field (Hu et al., 2018). We had left overwintering the ' $B X+$ ' and 'BX-' soil variants after conditioning them in summer and had observed the feedbacks still in the following spring. Prompted by this observation and motivated to evaluate our experimental approach, we wanted to answer, if ' $\mathrm{BX}+$ ' and ' $\mathrm{BX}-$ ' soil variants can be stored in a cold room at $4^{\circ} \mathrm{C}$ for at least one year without losing their efficacy. Similar to the field winter, we found that a 'technical' overwintering of the 'BX+' and 'BX-'soil variants in the cold room also preserved the BX-feedbacks (Figures 1 and 2, Data S1). This retention of feedback capacity has important practical consequences as there is no need to study BX-feedbacks immediately on freshly conditioned soils and that batches of conditioned soil can be stored and examined at later stages.

\section{3 | BX-feedbacks also function on wheat}

Like maize, wheat also produces BXs (Li et al., 2018; Niemeyer, 2009) and secretes them to the rhizosphere (Chen et al., 2010). Although a broad examination on how other or non-BX producing plant species such as for instance functional groups like legumes, forbs or crucifers deal with BX-conditioned soils would have been interesting, we chose to investigate wheat, as it is often following maize in crop rotations in Europe and because of agronomic relevance.

From our study, it is clear that BX-feedbacks, globally considering the biomass (Figure 3), chlorophyll and insect resistance measures (Figure 5), also function on wheat. Similar to maize, negative feedbacks on biomass were seen when wheat was grown on 'BX+' compared to 'BX-' soil variants (Figure 3, Data S2). Although not compared side-by-side, it appeared that BX-feedbacks on wheat growth tended to be more variable and/or weaker compared to maize. BX-feedbacks were also observed on wheat physiology (Figure S2 and Figure $5 \mathrm{~b}$ ) and insect resistance (Figure $5 \mathrm{a}$ ), but the direction of the feedbacks contrasted with maize responses to soil BX-conditioning. While the chlorophyll approximations were higher on ' $\mathrm{BX}+$ ' soil in the tested wheat cultivars, they were lower in maize (Hu et al., 2018). Opposite effects were also seen on insect performance with enhanced resistance of maize grown on 'BX+' soil (Hu et al., 2018), while the wheat cultivar Fiorina was more susceptible when grown on 'BX+' soil. At present such opposite BX feedback behaviours cannot be generalized for being either maize or wheat specific, not only because more cultivars per plant species would be required but mainly because both plant species exhibited strong genetic variation in their feedback responses (see below). 


\section{4 | Microbial and allelopathic components of BX- feedbacks}

'BX+ soils' contain both, BX chemicals and a thereby conditioned microbiota ('BX+ microbiota'), while the BX compounds are absent in ' $\mathrm{BX}$ - soils' and therefore, the microbiota is not conditioned by BXs ('BX - microbiota'). Thus, the differential feedbacks observed on plants have a microbial (BX+vs. BX- microbiota) and a chemical component (presence vs. absence of BXs). With our maize work, we had demonstrated the presence of this microbiota component using sterilization and complementation experiments (Hu et al., 2018). Sterilization did not affect the BX levels in BX+ soils, but abolished the feedbacks, which were restored with the addition of a microbial extracts to the sterilized and BX containing soils. We had formulated a model based on the presence of BXs and the microbiota presenting the drivers for the $\mathrm{BX}$ feedbacks. For the maize line $\mathrm{B} 73$, with which the sterilization and complementation experiments were performed, it is clear that feedbacks have a microbial and not an allelopathic component. Nevertheless, we explicitly include in the model the possibility of direct contributions of BX chemicals to the feedbacks, in particular, because BXs are renowned for their allelopathic effects on many plant species (Schandry \& Becker, 2020).

Because of the following two evidences, we speculate that the relative contributions of microbial and allelopathic components in BX feedbacks differ among response species or genotypes. For instance, the reductions in plant biomass of both maize (Figure 1) and wheat (Figure 3 ) intuitively suggest an allelopathic component in this feedback, as such effects of BX on cereals has been demonstrated (Acharya, Kaspar, \& Robertson, 2020). Nevertheless, for maize that grows on the maize conditioned $\mathrm{BX}+$ soils, the sterilization and complementation demonstrated clear a microbial and not an allelopathic one in the feedbacks (Hu et al., 2018). The previous conditioning of the soil by maize resulted in an enrichment of maize-adapted microbes in the $\mathrm{BX}+$ soils and an open question is how wheat responds to these maize-adapted microbes. Therefore, it remains to be shown for wheat whether the negative growth feedback is also consequence of microbial interactions or if this is not at least partly an allelopathic effect. Complementary experiments utilizing a BXdefective wheat mutant (currently not available) that produces $\mathrm{BX}+$ soils containing wheat-adapted microbes and performing reciprocal feedback experiments with maize would be necessary for understanding the role of microbial adaption in BX-feedbacks.

The second evidence, why we think of microbial and allelopathic components in BX feedbacks, is related to microbiota perception by the response plants. Maize plants growing on $\mathrm{BX}+$ soils exhibited elevated defence hormone levels and expression of defence marker genes that are reminiscent of ISR (Hu et al., 2018) and therefore, this point to a microbial component in the BX feedbacks. In contrast, wheat did not respond with altered defence hormone levels suggesting no microbial contribution to the feedbacks. This interpretation could be further corroborated with experiments testing for eventual microbiota differences of wheat when growing on maize conditioned $\mathrm{BX}+$ or $\mathrm{BX}-$ soils. As indicated above, the associations and thereby the feedbacks to a maize conditioned microbiota in $\mathrm{BX}+$ soils may differ between maize or wheat as host plants in the subsequent generation. A hypothesis is that the host plant in the next generation and the previously conditioned microbiota (here by maize) need to be adapted to each other to express a microbiota-induced systemic resistance.

Taken together, dedicated work related to microbiota adaptation and plant responsiveness is now needed to disentangle the microbial from allelopathic contributions to the observed BX-feedbacks.

\section{5 | Genetic variation in responsiveness to BX- feedbacks}

Insect resistance and chlorophyll content were unaffected in the cultivar Drifter, whereas Fiorina was more susceptible to the S. littoralis and had reduced chlorophyll levels when grown on 'BX+ soil' (Figure 5). Similar to the genotypic variation we found in the tested two wheat cultivars to BX-conditioned soils in this study, the two maize lines B73 and W22 expressed both the BX-dependent increased insect defences whereas growth suppression was only seen in B73 but not in W22 (Hu et al., 2018). Here, we now demonstrated that the genotype of the response plant explains the different growth feedbacks (Figure 5). It is not uncommon that different plant genotypes express differential responsiveness to microbes. Wheat cultivars respond differently to associations with beneficial microbes (Akbari, Gharanjik, Koobaz, \& Sadeghi, 2020; de León et al., 2020; Egamberdieva, 2010) as well as express different sensitivities to the allelopathic effect of BXs (Schulz, Marocco, Tabaglio, Macias, \& Molinillo, 2013). In the context of microbially triggered ISR, there is genetic variation in responsiveness as reported for barley (Shrestha et al., 2019). This study showed that an appropriate genetic background is required to perceive the microbial priming in order to express enhanced resistance. The common conclusion is obvious: there is genetic variation in host plant responsiveness to individual microbes or complex soil microbiomes as seen also in the BX feedbacks. The underlying mechanisms, how plants perceive a specific complex soil microbiota that induces systemic resistance or promoting growth, are not know. Ultimately, plant loci for positive responsiveness to microbiota feedbacks will open new opportunities to integrate beneficial plant-microbiome interactions into crop breeding programs.

\section{6 | Relevance of BX-feedbacks in agriculture}

Similar to Pineda et al. (2020), our studies on BX-feedbacks serve as evidence that the concept of PSFs may be exploited in cropping systems for pest control. There is often a discrepancy between results obtained in highly controlled greenhouse experiments and those in real-life field trials (Beals et al., 2020; Brinkman, Van der Putten, Bakker, \& Verhoeven, 2010; Forero, Grenzer, Heinze, Schittko, \& Kulmatiski, 2019). Nevertheless, several findings of our work under controlled conditions argue that these BX feedbacks may be relevant for agriculture: (a) BX-feedbacks were observed in natural field soils, 
(b) BX-feedbacks preserve their effectivity over a winter period and (c) agronomically relevant crops respond to BX-feedbacks. Field experiments with natural conditions and a rotation of wheat following maize are now necessary to answer the major emerging question from this work: do these BX-feedbacks have real-life agronomic implications?

\section{ACKNOWLEDGMENTS}

We thank Damian Amrein from the seed selection group (Agroscope) for providing the Fiorina wheat seeds. Special credits go to Nicolas Widmer, Pierre Pignon, Juerg Hiltbrunner and Fritz Kaeser (Agroscope) for field support and Philip Streckeisen (Agroscope) for technical support in the greenhouse. We also thank Diane Buerge (Agroscope) and Dr. Moritz Bigalke (Institute of Geography, University of Bern) for soil analyses. qPCR experiments were performed at the Genetic Diversity Centre (GDC), ETH Zurich.

\section{CONFLICT OF INTEREST}

The authors declare no conflicts of interest.

\section{AUTHOR CONTRIBUTIONS}

Klaus Schlaeppi, Matthias Erb and Marcel G. A. van der Heijden conceived the original project and Klaus Schlaeppi supervised the experiments. Andrea Sánchez-Vallet, Daniel Croll, Matthias Erb and Marcel G. A. van der Heijden provided field resources, laboratory infrastructure and seeds. Selma Cadot, Valentin Gfeller and Lingfei Hu performed the experiments with support by Gaétan Glauser for analytical work, by Nikhil Singh for Zymoseptoria work and support by Andrea Sánchez-Vallet for qPCR analysis. Selma Cadot and Klaus Schlaeppi analysed the data. Selma Cadot and Klaus Schlaeppi wrote the manuscript with input from all authors.

\section{DATA AVAILABILITY STATEMENT}

The datasets generated and analysed during the current study are available in the 'Cadot_et_al_wheat_feedbacks' repository (https:// github.com/PMI-Basel).

\section{ORCID}

Selma Cadot (D) https://orcid.org/0000-0003-3939-2593

Valentin Gfeller (D) https://orcid.org/0000-0001-8896-7280

Lingfei Hu (D) https://orcid.org/0000-0002-7791-9440

Andrea Sánchez-Vallet (D) https://orcid.org/0000-0002-3668-9503

Gaétan Glauser (iD https://orcid.org/0000-0002-0983-8614

Daniel Croll ID https://orcid.org/0000-0002-2072-380X

Matthias Erb (D) https://orcid.org/0000-0002-4446-9834

Marcel G. A. van der Heijden (D) https://orcid.org/0000-0001-7040-

1924

Klaus Schlaeppi (D) https://orcid.org/0000-0003-3620-0875

\section{REFERENCES}

Acharya, J., Kaspar, T. C., \& Robertson, A. E. (2021). Effect of 6-Methoxy2-Benzoxazolinone (MBOA) on pythium species and corn seedling growth and disease. Plant Disease, 105(4), 752-757. https://doi.org/ 10.1094/pdis-04-20-0824-sc
Akbari, A., Gharanjik, S., Koobaz, P., \& Sadeghi, A. (2020). Plant growth promoting Streptomyces strains are selectively interacting with the wheat cultivars especially in saline conditions. Heliyon, 6, e03445.

Bakker, P. A. H. M., Pieterse, C. M. J., de Jonge, R., \& Berendsen, R. L. (2018). The soil-borne legacy. Cell, 172, 1178-1180.

Barrett, L. G., Zala, M., Mikaberidze, A., Alassimone, J., Ahmad, M., McDonald, B. A., \& Sánchez-Vallet, A. (2021). Mixed infections alter transmission potential in a fungal plant pathogen. Environmental Microbiology, 23(4), 2315-2330.

Bates, D., Mächler, M., Bolker, B., \& Walker, S. (2015). Fitting linear mixedeffects models using Ime4. Journal of Statistical Software, 67, 1-48.

Beals, K. K., Moore, J. A. M., Kivlin, S. N., Bayliss, S. L. J., Lumibao, C. Y., Moorhead, L. C., ... Schweitzer, J. A. (2020). Predicting plant-soil feedback in the field: Meta-analysis reveals that competition and environmental stress differentially influence PSF. Frontiers in Ecology and Evolution, 8, 191.

Bennett, J. A., Maherali, H., Reinhart, K. O., Lekberg, Y., Hart, M. M., \& Klironomos, J. (2017). Plant-soil feedbacks and mycorrhizal type influence temperate forest population dynamics. Science, 355, 181-184.

Berendsen, R. L., Pieterse, C. M. J., \& Bakker, P. A. H. M. (2012). The rhizosphere microbiome and plant health. Trends in Plant Science, 17, 478-486.

Berendsen, R. L., Vismans, G., Yu, K., Song, Y., de Jonge, R., Burgman, W. P., ... Pieterse, C. M. J. (2018). Disease-induced assemblage of a plant-beneficial bacterial consortium. The ISME Journal, 12, 1496-1507.

Bergmann, J., Verbruggen, E., Heinze, J., Xiang, D., Chen, B., Joshi, J., \& Rillig, M. C. (2016). The interplay between soil structure, roots, and microbiota as a determinant of plant-soil feedback. Ecology and Evolution, 6, 7633-7644.

Bever, J. D. (2003). Soil community feedback and the coexistence of competitors: Conceptual frameworks and empirical tests. New Phytologist, 157, 465-473.

Bever, J. D., Platt, T. G., \& Morton, E. R. (2012). Microbial population and community dynamics on plant roots and their feedbacks on plant communities. Annual Review of Microbiology, 66, 265-283.

Bezemer, T. M., Lawson, C. S., Hedlund, K., Edwards, A. R., Brook, A. J., Igual, J. M., ... Van Der Putten, W. H. (2006). Plant species and functional group effects on abiotic and microbial soil properties and plant-soil feedback responses in two grasslands. Journal of Ecology, 94, 893-904.

Brennan, R. J. B., Glaze-Corcoran, S., Wick, R., \& Hashemi, M. (2020). Biofumigation: An alternative strategy for the control of plant parasitic nematodes. Journal of Integrative Agriculture, 19, 1680-1690.

Brinkman, E. P., Van der Putten, W. H., Bakker, E.-J., \& Verhoeven, K. J. F. (2010). Plant-soil feedback: Experimental approaches, statistical analyses and ecological interpretations. Journal of Ecology, 98, 1063-1073.

Cadot, S., Guan, H., Bigalke, M., Walser, J.-C., Jander, G., Erb, M., van der Heijden, M. G. A., \& Schlaeppi, K. (2021). Specific and conserved patterns of microbiota-structuring by maize benzoxazinoids in the field. Microbiome, 9(1). http://dx.doi.org/10.1186/s40168-021-01049-2

Chen, K.-J., Zheng, Y.-Q., Kong, C.-H., Zhang, S.-Z., Li, J., \& Liu, X.-G. (2010). 2,4-Dihydroxy-7-methoxy-1,4-benzoxazin-3-one (DIMBOA) and 6-Methoxy-benzoxazolin-2-one (MBOA) Levels in the Wheat Rhizosphere and Their Effect on the Soil Microbial Community Structure. Journal of Agricultural and Food Chemistry, 58, 12710-12716.

Cotton, T. E. A., Pétriacq, P., Cameron, D. D., Meselmani, M. A., Schwarzenbacher, R., Rolfe, S. A., \& Ton, J. (2019). Metabolic regulation of the maize rhizobiome by benzoxazinoids. The ISME Journal, 13, 1647-1658.

de León, D. G., Vahter, T., Zobel, M., Koppel, M., Edesi, L., Davison, J., ... Öpik, M. (2020). Different wheat cultivars exhibit variable responses to inoculation with arbuscular mycorrhizal fungi from organic and conventional farms. PLoS One, 15, e0233878.

Egamberdieva, D. (2010). Growth response of wheat cultivars to bacterial inoculation in calcareous soil. Plant, Soil and Environment, 56, 570-573. 
Erb, M., Balmer, D., De Lange, E. S., Von Merey, G., Planchamp, C. Robert, C. A. M., et al. (2011). Synergies and trade-offs between insect and pathogen resistance in maize leaves and roots. Plant, Cell \& Environment, 34, 1088-1103.

Fahrmeir, L., Kneib, T., Lang, S., \& Marx, B. (2013). Regression: Models, methods and applications. Berlin Heidelberg: Springer-Verlag.

Forero, L. E., Grenzer, J., Heinze, J., Schittko, C., \& Kulmatiski, A. (2019). Greenhouse- and field-measured plant-soil feedbacks are not correlated. Frontiers in Environmental Science, 7, 184.

Gimsing, A. L., \& Kirkegaard, J. A. (2009). Glucosinolates and biofumigation: Fate of glucosinolates and their hydrolysis products in soil. Phytochemistry Reviews, 8, 299-310.

Glauser, G., Vallat, A., \& Balmer, D. (2014). Hormone profiling. Methods in Molecular Biology, 1062, 597-608.

Hannula, S. E., Ma, H., Pérez-Jaramillo, J. E., Pineda, A., \& Bezemer, T. M. (2020). Structure and ecological function of the soil microbiome affecting plant-soil feedbacks in the presence of a soil-borne pathogen. Environmental Microbiology, 22, 660-676.

Heinen, R., Biere, A., \& Bezemer, T. M. (2020). Plant traits shape soil legacy effects on individual plant-insect interactions. Oikos, 129, 261-273.

Hu, L., Robert, C. A. M., Cadot, S., Zhang, X., Ye, M., Li, B., ... Erb, M. (2018). Root exudate metabolites drive plant-soil feedbacks on growth and defense by shaping the rhizosphere microbiota. Nature Communications, 9, 2738.

Huberty, M., Choi, Y. H., Heinen, R., \& Bezemer, T. M. (2020). Aboveground plant metabolomic responses to plant-soil feedbacks and herbivory. Journal of Ecology, 108, 1703-1712.

Inderjit, \& Duke, S. O. (2003). Ecophysiological aspects of allelopathy. Planta, 217, 529-539.

Jones, D. L., \& Darrah, P. R. (1995). Influx and efflux of organic acids across the soil-root interface of Zea mays $\mathrm{L}$. and its implications in rhizosphere C flow. Plant and Soil, 173, 103-109.

Kudjordjie, E. N., Sapkota, R., Steffensen, S. K., Fomsgaard, I. S., \& Nicolaisen, M. (2019). Maize synthesized benzoxazinoids affect the host associated microbiome. Microbiome, 7, 59.

Kutáková, E., Herben, T., \& Münzbergová, Z. (2018). Heterospecific plantsoil feedback and its relationship to plant traits, species relatedness, and co-occurrence in natural communities. Oecologia, 187, 679-688.

Li, B., Förster, C., Robert, C. A. M., Züst, T., Hu, L., Machado, R. A. R., ... Erb, M. (2018). Convergent evolution of a metabolic switch between aphid and caterpillar resistance in cereals. Science Advances, 4, eaat6797.

Maag, D., Dalvit, C., Thevenet, D., Köhler, A., Wouters, F. C., Vassão, D. G., ... Glauser, G. (2014). 3- $\beta$-d-Glucopyranosyl-6-methoxy2-benzoxazolinone (MBOA-N-Glc) is an insect detoxification product of maize 1,4-benzoxazin-3-ones. Phytochemistry, 102, 97-105.

Macías, F. A., Marín, D., Oliveros-Bastidas, A., \& Molinillo, J. M. G. (2009). Rediscovering the bioactivity and ecological role of 1,4-benzoxazinones. Natural Product Reports, 26, 478-489.

Mariotte, P., Mehrabi, Z., Bezemer, T. M., De Deyn, G. B., Kulmatiski, A., Drigo, B., ... Kardol, P. (2018). Plant-soil feedback: Bridging natural and agricultural sciences. Trends in Ecology \& Evolution, 33, 129-142.

Martyniuk, S., Stochmal, A., Macías, F. A., Marín, D., \& Oleszek, W. (2006). Effects of some benzoxazinoids on in vitro growth of Cephalosporium gramineum and other fungi pathogenic to cereals and on Cephalosporium stripe of winter wheat. Journal of Agricultural and Food Chemistry, 54, 1036-1039.

Meile, L., Croll, D., Brunner, P. C., Plissonneau, C., Hartmann, F. E., McDonald, B. A., \& Sánchez-Vallet, A. (2018). A fungal avirulence factor encoded in a highly plastic genomic region triggers partial resistance to septoria tritici blotch. The New Phytologist, 219, 1048-1061.

Neal, A. L., Ahmad, S., Gordon-Weeks, R., \& Ton, J. (2012). Benzoxazinoids in root exudates of maize attract pseudomonas putida to the rhizosphere. PLoS One, 7, e35498.

Neal, A. L., \& Ton, J. (2013). Systemic defense priming by Pseudomonas putida KT2440 in maize depends on benzoxazinoid exudation from the roots. Plant Signaling \& Behavior, 8, e22655.
Niemeyer, H. M. (2009). Hydroxamic acids derived from 2-hydroxy-2h-1,4-benzoxazin-3(4H)-one: Key defense chemicals of cereals. Journal of Agricultural and Food Chemistry, 57, 1677-1696.

Pieterse, C. M. J., Zamioudis, C., Berendsen, R. L., Weller, D. M., Van Wees, S. C. M., \& Bakker, P. A. H. M. (2014). Induced systemic resistance by beneficial microbes. Annual Review of Phytopathology, 52, 347-375.

Pineda, A., Kaplan, I., Hannula, S. E., Ghanem, W., \& Bezemer, T. M. (2020). Conditioning the soil microbiome through plant-soil feedbacks suppresses an aboveground insect pest. New Phytologist, 226, 595-608.

Pizano, C., Kitajima, K., Graham, J. H., \& Mangan, S. A. (2019). Negative plant-soil feedbacks are stronger in agricultural habitats than in forest fragments in the tropical Andes. Ecology, 100, e02850.

Poveda, J., Eugui, D., \& Velasco, P. (2020). Natural control of plant pathogens through glucosinolates: An effective strategy against fungi and oomycetes. Phytochemistry Reviews, 19, 1045-1059.

R Development Core Team. (2017). R: A language and environment for statistical computing. Vienna, Austria: R Foundation for Statistical Computing.

Revillini, D., Gehring, C. A., \& Johnson, N. C. (2016). The role of locally adapted mycorrhizas and rhizobacteria in plant-soil feedback systems. Functional Ecology, 30, 1086-1098.

Sasse, J., Martinoia, E., \& Northen, T. (2018). Feed your friends: Do plant exudates shape the root microbiome? Trends in Plant Science, 23, 25-41.

Schalchli, H., Pardo, F., Hormazábal, E., Palma, R., Guerrero, J., \& Bensch, E. (2012). Antifungal activity of wheat root exudate extracts on Gaeumannomyces graminis var. Tritici growth. Journal of Soil Science and Plant Nutrition, 12, 329-337.

Schandry, N., \& Becker, C. (2020). Allelopathic plants: Models for studying plant-Interkingdom interactions. Trends in Plant Science, 25, 176-185.

Schulz, M., Marocco, A., Tabaglio, V., Macias, F. A., \& Molinillo, J. M. G. (2013). Benzoxazinoids in rye allelopathy - from discovery to application in sustainable weed control and organic farming. Journal of Chemical Ecology, 39, 154-174.

Shrestha, A., Elhady, A., Adss, S., Wehner, G., Böttcher, C., Heuer, H., ... Schikora, A. (2019). Genetic differences in barley govern the responsiveness to $\mathrm{N}$-acyl homoserine lactone. Phytobiomes Journal, 3, 191-202.

Sicker, D., Frey, M., Schulz, M., \& Gierl, A. (2000). Role of natural benzoxazinones in the survival strategy of plants. International Review of Cytology, 198, 319-346.

Singh, N. K., Badet, T., Abraham, L., \& Croll, D. (2021). Rapid sequence evolution driven by transposable elements at a virulence locus in a fungal wheat pathogen. BMC Genomics, 22(1). http://dx.doi.org/10. 1186/s12864-021-07691-2

Smith-Ramesh, L. M., \& Reynolds, H. L. (2017). The next frontier of plantsoil feedback research: Unraveling context dependence across biotic and abiotic gradients. Journal of Vegetation Science, 28, 484-494.

Stringlis, I. A., Yu, K., Feussner, K., de Jonge, R., Van Bentum, S., Van Verk, M. C., ... Pieterse, C. M. J. (2018). MYB72-dependent coumarin exudation shapes root microbiome assembly to promote plant health. Proceedings of the National Academy of Sciences, 115, E5213-E5222.

Teste, F. P., Kardol, P., Turner, B. L., Wardle, D. A., Zemunik, G., Renton, M., \& Laliberté, E. (2017). Plant-soil feedback and the maintenance of diversity in Mediterranean-climate shrublands. Science, 355, 173-176.

van der Putten, W. H. (2017). Belowground drivers of plant diversity. Science, 355, 134-135.

van der Putten, W. H., Bardgett, R. D., Bever, J. D., Bezemer, T. M., Casper, B. B., Fukami, T., ... Wardle, D. A. (2013). Plant-soil feedbacks: The past, the present and future challenges. Journal of Ecology, 101, 265-276.

Wagg, C., Boller, B., Schneider, S., Widmer, F., \& van der Heijden, M. G. A. (2015). Intraspecific and intergenerational differences in plant-soil feedbacks. Oikos, 124, 994-1004.

Wickham, H. (2016). ggplot2: Elegant graphics for data analysis. New York, NY: Springer-Verlag.

Wouters, F. C., Blanchette, B., Gershenzon, J., \& Vassão, D. G. (2016). Plant defense and herbivore counter-defense: 
Benzoxazinoids and insect herbivores. Phytochemistry Reviews, 15, 1127-1151.

Yuan, J., Zhao, J., Wen, T., Zhao, M., Li, R., Goossens, P., ... Shen, Q. (2018). Root exudates drive the soil-borne legacy of aboveground pathogen infection. Microbiome, 6, 156.

Zhao, M., Zhao, J., Yuan, J., Hale, L., Wen, T., Huang, Q., ... Shen, Q. (2021). Root exudates drive soil-microbe-nutrient feedbacks in response to plant growth. Plant, Cell \& Environment, 44, 613-628.

Zhou, S., Richter, A., \& Jander, G. (2018). Beyond defense: Multiple functions of benzoxazinoids in maize metabolism. Plant and Cell Physiology, 59, 1528-1537.

Zhu, F., Heinen, R., van der Sluijs, M., Raaijmakers, C., Biere, A., \& Bezemer, T. M. (2018). Species-specific plant-soil feedbacks alter herbivore-induced gene expression and defense chemistry in Plantago lanceolata. Oecologia, 188, 801-811.

\section{SUPPORTING INFORMATION}

Additional supporting information may be found in the online version of the article at the publisher's website.

How to cite this article: Cadot, S., Gfeller, V., Hu, L., Singh, N., Sánchez-Vallet, A., Glauser, G., Croll, D., Erb, M., van

der Heijden, M. G. A., \& Schlaeppi, K. (2021). Soil composition and plant genotype determine benzoxazinoid-mediated plantsoil feedbacks in cereals. Plant, Cell \& Environment, 1-13.

https://doi.org/10.1111/pce.14184 